\title{
Alexander der Große und der indische Raja Puru: Zur Exotik in einem Libretto Metastasios und in darauf basierenden Opern von Hasse und Händel ${ }^{1}$
}

\author{
Ralph P. Locke
}

Für Jürgen Thym

Im Verlauf der letzten fünf Jahrhunderte bedienten sich westliche Musiker einer breiten Palette musikalischer Gattungen, um Vorstellungen von exotischen Ländern und Völkern zu gestalten: von Volksliedern über abendfüllende Opern und Oratorien bis zu für die Laute komponierten fremdländischen Tänzen und heutigen Broadway-Musicals, ganz zu schweigen von der Musik für Filme und Videospiele. In den bisherigen Diskussionen darüber, wie das Exotische musikalisch evoziert wurde, blieben jedoch viele Opern des 17. und 18. Jahrhunderts unberücksichtigt, in denen nicht-europäische Hauptfiguren auftreten. ${ }^{2}$ Vorliegende Studie soll dazu beitragen, die Erforschung des Exotischen in der westlichen Musik in eine neue Richtung zu lenken, indem Pietro Metastasios Alessandro nell'Indie, das Libretto einer Opera seria von 1729, deren Handlung auf dem indischen Subkontinent spielt, sowie zwei auf diesem Libretto basierende Opern, Händels Poro re dell'Indie (1731) und Hasses Cleofide (1731), untersucht werden.

1 Der vorliegende Beitrag ist eine etwas gekürzte Version meines Essays „Alexander the Great and the Indian Raja Puru: Exoticism in a Metastasio Libretto as Set by Hasse and by Handel“", der demnächst in einer englischsprachigen Zeitschrift erscheinen wird. Beide Versionen sind Erweiterungen einiger Abschnitte aus meinem jüngsten Buch Music and the Exotic from the Renaissance to Mozart und wiederum anderer Absätze aus folgendem Artikel: Ralph P. Locke: Alien Adventures: Exoticism in Italian-Language Operas of the Baroque. In: Musical Times 150, Nr. 1909, 53-69. Eine kürzere Version des Artikels aus der Musical Times, übersetzt von Arnold Jacobshagen, erschien als: Ralph P. Locke: Exotismus in der Opera seria? In: Arnold Jacobshagen und Panja Mücke (Hg.): Händels Opern: Das Handbuch. Laaber 2009, Teilbd. 1, 221-235. Mein Dank gilt Rebekah Ahrendt, Lorenzo Bianconi, Margaret R. Butler, Regina Compton, Roger Freitas, Ellen T. Harris, Richard G. King, Nathan Link, Patrick Macey und Jürgen Thym, die so freundlich waren, Entwürfe dieser Studie gegenzulesen. Gewidmet sei dieser Beitrag Jürgen Thym, dem kenntnisreichen Wissenschaftler, gütigen Kollegen und Mitstreiter, der in hingebungsvoller Weise die Geschichte der Musikwissenschaft innerhalb der Eastman School of Music (University of Rochester ) - einer der musikalischen Praxis gewidmeten Musikhochschule - gestaltete.

2 Andere wichtige Gattungen, die ebenfalls oft aus der Diskussion um Musik und das Exotische ausgespart werden, sind das Bänkellied (Henry Howards Lied über drei CherokeeHäuptlinge, um 1762), das Ballet de cour (zum Beispiel La délivrance de Renaud, 1617), das dramatische Oratorium (zum Beispiel Händels Belshazzar, 1745) und die Opéra comique aus der Zeit von Lesage (Arlequin sultane favorite, 1715). Zu den erwähnten Werken lege ich in meinem Buch (Ralph P. Locke: Music and the Exotic from the Renaissance to Mozart. Cambridge 2015) Fallstudien vor. 


\section{Exotismus obne exotischen Stil}

Die bisherige Forschung hat Opern mit, exotischen' Figuren aus der Zeit vor 1750 vor allem aus zwei Gründen nicht berücksichtigt. Erstens sind fast alle Opern nach der Entstehung dieser Gattung um 1600 bis zur Mitte des 18. Jahrhunderts innerhalb weniger Jahre oder Jahrzehnte aus dem Repertoire verschwunden, selbst wenn sie bei Erstaufführung großen Beifall erhielten. Glücklicherweise fanden einige dieser Opern im 20. Jahrhundert ein neues Publikum. Dieser Trend der Wiederentdeckung hat sich in den letzten Jahrzehnten noch verstärkt, auch dank einfallsreicher und stilistisch feinfühliger Sänger und Instrumentalisten wie der Mezzosopranistin Gloria Banditelli, dem Countertenor Andreas Scholl und dem Cembalospieler und Dirigenten Christophe Rousset. Zweitens: Selbst wenn ein solches Werk kompetent aufgeführt wird, lassen sich Publikum, Kritiker und Fachleute oft von modernen Annahmen darüber leiten, auf welche Weise Musik Vorstellungen von Exotik evozieren kann. Insbesondere wird oft nach Spuren einer ,exotischen Färbung' gesucht: musikalische Zeichen oder Codes der Andersartigkeit. Tatsächlich nehmen wir oft an, dass bestimmte musikalische Codes notwendige und hinreichende Bedingungen für Exotik sind, weil im Musiktheater seit dem späten 18. Jahrhundert bestimmte Stilelemente an prominenter Stelle verwendet wurden, die Komponisten und Publikum als Hinweise auf exotische Regionen interpretierten. $\mathrm{Zu}$ den oft untersuchten Beispielen gehören Mozarts Die Entführung aus dem Serail (1782), die auf effektive Weise den Janitscharen-Stil einsetzt (auch als Alla turca-Stil bekannt). Auch Puccinis Madama Butterfly (1904-1907) verwendet viele japanische und - wie neuere Forschungen ergeben haben - chinesische Melodien, integriert aber auch Grundelemente, die eindeutig dazu dienen sollten, ein imaginäres Japan zu evozieren, zum Beispiel die Pentatonik und die prominente Rolle der Harfe als Verweis auf die Koto. ${ }^{3}$

In meinen Büchern Musical Exoticism: Images and Reflections (2009) und Music and the Exotic from the Renaissance to Mozart (2015) bezeichne ich die Annahme, dass musikalischer Exotismus die Anwesenheit von eindeutig fremden - oder zumindest fremd wirkenden - musikalischen Stilmerkmalen voraussetzt, als das Paradigma des exotischen Stils:

Das ,Paradigma des exotischen Stils' identifiziert eine exotisierende Absicht oder Botschaft in einer Passage oder einem ganzen Werk, wenn (und nur wenn) stilistische Mittel

$3 \mathrm{Zu}$ exotisierenden Konzeptionen und Merkmalen in Mozarts Entführung siehe Locke: Music and the Exotic (Anm. 2), 26-27, 308-313. Ralph P. Locke: Musical Exoticism: Images and Reflections. Cambridge 2009, 114-123. Für eine ähnliche Analyse von Puccinis Madama Butterfly siehe ebd., 175-213. W. Anthony Sheppard hat 2012 herausgefunden, dass zwei wichtige Motive in Madama Butterfly chinesische Ursprünge haben; siehe W. Anthony Sheppard: Music Box as Muse to Puccini's ,Butterfly'. In: The Nerw York Times, 15. Juni 2012, http://www.nytimes.com/2012/06/17/arts/music/puccini-opera-echoes-a-music-boxat-the-morris-museum.html?pagewanted=all\&_r=1, 29. März 2016. W. Anthony Sheppard: Puccini and the Music Boxes. In: Journal of the Royal Musical Association 140, 2015, 41-92. 
verwendet werden, die (1) von zu diesem Zeitpunkt vorherrschenden Normen abweichen und (2) entweder von den musikalischen Traditionen eines dargestellten exotischen Ortes abgeleitet sind oder irgendwie als Hinweise auf diesen Ort verstanden werden können (zum Beispiel durch einen erfundenen Stil oder durch beabsichtigte Andeutungen von Fremdheit). ${ }^{4}$

Bezogen auf Werke wie die oben erwähnten Opern von Mozart und Puccini ist das Paradigma des exotischen Stils äußerst aufschlussreich, während es bei Werken aus der Zeit vor der Mitte des 18. Jahrhunderts oft nicht greift. Wenn zum Beispiel eine Opera seria aus dem frühen 18. Jahrhundert eine nicht-westliche Kultur oder nicht-westliche Personen darstellt, dann werden nur selten stilistische Elemente verwendet, die sich erkennbar von denen unterscheiden, die der Komponist verwendet hätte, spielte das Werk irgendwo in Europa und träten nur europäische Figuren auf, zum Beispiel Griechen oder Römer.

In einem Werk aus der Zeit vor der Mitte des 18. Jahrhunderts wurden Merkmale eines nicht-westlichen Schauplatzes, einer ethnischen Gruppe oder eines exotischen Individuums zum größten Teil durch nicht-musikalische Mittel inszeniert. Dazu gehörten etwa die Namen der Protagonisten, Details der Handlung und der dramatischen Darbietung auf der Bühne (insbesondere das Verhalten und die Reaktionen einer Figur), der genaue Text (Erklärungen, Rechtfertigungen), der von einer bestimmten Figur gesungen wird, und vielleicht einige ungewöhnliche Merkmale des Bühnenbilds und der Kostüme (wie etwa eine gemalte Kulisse, die auf einem Reisebericht basiert, oder ein mit gefedertem Turban ausgestatteter türkischer oder persischer Herrscher). ${ }^{5}$

Die Komponisten verstärkten diese Charakteristika oft durch die Musik, aber nicht indem sie musikalische Klänge und Stilmittel verwendeten, die irgendwie ,fremd' klingen. Stattdessen setzten sie verschiedene normative Stilmittel ein, die Komponisten von Werken des Musiktheaters dieser Zeit zur Verfügung standen, um dem Gefühl des gesungenen Textes zu entsprechen. Mit anderen Worten: Die Absicht des Komponisten war es, die musikalische Begleitung eines bestimmten Moments innerhalb eines solchen Werks den Gefühlen, dem Verhalten sowie den aktuellen und zukünftigen Handlungen der jeweils singenden Figuren anzupassen. Wird ein persischer oder mongolischer Krieger zum Beispiel als leicht reizbar und aufbrausend gezeigt - eine von mehreren Eigenschaften, die besonders nichtwestlichen männlichen Herrschern, Kriegern und religiösen Autoritäten zugeschrieben wurde -, dann werden diese Gefühle durch die musikalischen Mittel dargestellt, mit denen die Komponisten üblicherweise den Eindruck von Zorn ver-

4 Locke: Music and the Exotic (Anm. 2), 17-19. Vgl. ebd., 42-59. Die beiden Paradigmen wurden zum ersten Mal in folgendem Aufsatz erläutert: Ralph P. Locke: A Broader View of Musical Exoticism. In: Journal of Musicology 24, 2007, 477-521.

5 Für Beispiele der Figurenzeichnung und des Bühnenbilds in Händels Opern, die sich auf Berichte von Reisenden aus dem Osten beziehen (zum Beispiel Aaron Hill, der den Entwurf für das Libretto für Rinaldo verfasst hat) vgl. Ellen T. Harris: George Frideric Handel: A Life with Friends. New York 2014, 107-113. 
stärken: zum Beispiel dynamische Sprünge in der Gesangslinie oder eindringliche rhythmische Figuren in der Orchesterbegleitung. Solche Mittel trugen in der Oper der Zeit dazu bei, einen nicht-westlichen von einem westlichen Mann zu unterscheiden - obwohl es auch hier wieder namhafte Ausnahmen gibt. Aber solche Elemente erfüllen ihre Charakterisierungsfunktion, ohne dabei auf einen ,exotischen' Musikstil zurückzugreifen - ja sogar ohne notwendigerweise überhaupt stilistischen Besonderheiten einzuführen. Aus diesem Grund ist es meist nicht hilfreich, das Paradigma des exotischen Stils auf Opern zu übertragen, die vor dem Aufkommen des Alla turca-Stils in der Mitte bis Ende des 18. Jahrhunderts entstanden.

Daher habe ich ein zweites, weiter gefasstes Paradigma zum Verständnis des musikalischen Exotismus vorgeschlagen. Es berücksichtigt zwar den Aspekt der ,exotischen Färbung' des Stils, bezieht aber auch viele andere Aspekte ein, zum Beispiel den strategischen Gebrauch von eher konventionellen Charakterisierungsmitteln. Ich nenne dies das Paradigma der vollständig kontextualisierten Musik. Da das weiter gefasste Paradigma deutlich mehr Faktoren in die Untersuchung einbezieht, können auch Werke analysiert werden, die das Paradigma des exotischen Stils nicht erklären kann. Ich fasse das weiter angelegte Paradigma wie folgt zusammen:

Das ,Paradigma der vollständig kontextualisierten Musik` geht davon aus, dass bei der Repräsentation des Exotischen in der Musik potenziell jede Art von musikalischen Mitteln eingesetzt wird, inklusive jener, die zu einer bestimmten Zeit die stilistische Norm bildeten. In vielen Vokalwerken und Werken des Musiktheaters verdeutlichen die Worte den Ort (zum Beispiel die ethnische Identität einer Opernfigur), wodurch der Komponist die Freiheit gewinnt, eine breitere Palette von möglichen Verfahren anzuwenden, um diese Darstellung zu intensivieren. Der erste Teil des Paradigmas - ,die ganze Musik - erinnert uns daran, dass der Rezipient ruhig alle musikalischen Elemente und Passagen in einem Stück einbeziehen sollte, inklusive all jener, die unter das Paradigma des exotischen Stils fallen. Der zweite Teil, der auf den Kontext der Musik Bezug nimmt, kann sowohl Grundelemente der Gattung enthalten, als auch nicht-musikalische Konzepte - wie etwa kulturelle Stereotypen, die zur damaligen Zeit als gegeben angenommen wurden: zum Beispiel weit verbreitete Vorstellungen über türkische Sultane, türkische Haremsfrauen, nordamerikanische ,Indianerhäuptlinge ${ }^{6}$ oder chinesische Minister. Folglich berücksichtigt das Paradigma der vollständig kontextualisierten Musik auch die nicht-musikalischen Elementen wie den gesungenen Text, die Regieanweisungen, das Kostümbild, aber auch die kulturellen Einstellungen, die auf der Metaebene des Werkes liegen. ${ }^{6}$

Der vorliegende Beitrag illustriert die Vorzüge eines solchen weiter gefassten Paradigmas, indem er eines der am häufigsten vertonten Libretti des 18. Jahrhunderts und zwei seiner besonders erfolgreichen Vertonungen untersucht. Bei dem Libret-

6 Locke: Music and the Exotic (Anm. 2), 20-21. Vgl. Locke: Musical Exoticism (Anm. 3), 59-71. Einige andere Forscher haben die Notwendigkeit eines weiter gefassten Ansatzes erkannt. Harris hat überzeugend dargelegt, dass Händel in den West/Ost-Opern manchmal reiche und ungewöhnliche Klangmittel verwendete, um den östlichen Luxus zu porträtieren (zum Beispiel Cleopatra in Alexander Balus), aber dass er solche Klänge öfter den westlichen Figuren zugewiesen hat (zum Beispiel in Rinaldo und Riccardo Primo), um die größere Stärke und Tugend der Westler anzudeuten (Harris: George Frideric Handel [Anm. 5], 77-85, 103-107). 
to handelt es sich um Pietro Metastasios Alessandro nell'Indie (Alexander der Große in Indien). Dieser kunstvolle Bühnentext wurde 1729 in einer Vertonung des angesehenen Komponisten Leonardo Vinci in Neapel uraufgeführt. Danach wurde er von zahlreichen Komponisten in Musik gesetzt - die Forschung hat 71 Vertonungen zusammengestellt -, wobei einige dieser Versionen viele Arien oder Rezitativpassagen herausgestrichen oder neue hinzufügt haben. Im Folgenden sollen zwei lose auf Metastasios Text basierende Opern genauer betrachtet werden: Georg Friedrich Händels Poro re dell'Indie und Johann Adolf Hasses Cleofide. Beide Opern wurden 1731 uraufgeführt: Händels Oper in einem öffentlichen Opernhaus in London, dem King's Theatre; Hasses Version mit einer stärker überarbeiteten Fassung des Librettos am Sächsischen Hof in Dresden. Die Gegenüberstellung und der Vergleich dieser beiden Vertonungen soll verdeutlichen, wie Nicht-Europäer von Komponisten italienischsprachiger Opera seria im 18. Jahrhundert dargestellt wurden.

\section{Stereotype des Ostens und die griechisch-römische moralische Norm}

Die beiden männlichen Protagonisten in Metastasios Libretto Alessandro nell'Indie basieren auf historischen Personen: Alexander der Große und Puru, ${ }^{7}$ ein berühmter Raja aus Paurava, einer Region im Punjab. Die Elefanten von Purus Armee wurden 326 v. Chr. am linken Ufer des Hydaspes von den Reitern der Griechen besiegt. ${ }^{8}$ Metastasios Libretto greift Elemente aus mehreren älteren literarischen Versionen auf, darunter Racines Theaterstück Alexandre le Grand (1665). Dort hat jeder der beiden Herrscher einen Armeegeneral (Timagene für Alessandro, Gandarte für Poro). Auch die von Alexander und Poro umworbene weibliche Hauptfigur Metastasios ist in den antiken Quellen bezeugt: Cleofide ist die Herrscherin über die indische Region, die nur durch einen Grenzfluss von Poros Gebiet getrennt ist. ${ }^{9}$ Metastasio war wahrscheinlich glücklich, einen Namen verwenden zu können, der eine etwas spätere ,Kleo' anklingen lässt: die Königin Kleopatra von Ägypten, der bekannte Prototyp der östlichen Frau, die gleichermaßen schön und politisch versiert war. ${ }^{10}$

7 In den griechischen Quellen wird er ,Poros` genannt und in den römischen ,Porus'. In Metastasios Libretto wurden beide Namen zu ,Alessandro' und ,Poro' italienisiert.

8 Dieser Fluss ist auch unter dem Namen ,Jhelam‘ bekannt und verläuft fast vollständig im heutigen Pakistan.

9 Kleophis (vermutlich abgeleitet vom sanskritischen Namen ,Kripa ${ }^{`}$ ) war laut verschiedenen griechischen und römischen Schriftstellern die Frau oder Mutter des Kriegsfürsten der Assakener, einem wichtigen Reitervolk im heutigen Peshawar-Tal. Einige der antiken Quellen behaupten, sie habe versucht, den griechischen Eroberer Alexander durch Geschenke und sogar Liebe zu besänftigen; die beiden römischen Autoren Curtius und Justinus berichten, sie habe ihm einen Sohn geboren.

10 Lucy Hughes-Hallett: Cleopatra: Histories, Dreams and Distortions. New York 1990. 
Thomas Betzwieser zufolge beschränke sich der Exotismus in vielen Opern zu Metastasios Alessandro-Libretto lediglich auf die oberflächliche „Topografie“, da die Handlung zwar in Indien spiele, ${ }^{11}$ doch das ferne Land einfach als Schauplatz für eine herkömmliche Opera seria diene. Tatsächlich aber evoziert Metastasios Libretto lokalspezifische Besonderheiten auf mannigfaltige Art und Weise, etwa durch die Namen der indischen Figuren, ${ }^{12}$ durch das konkrete Verhalten dieser Protagonisten und durch die Worte, die sie singen (Gefühle, Reaktionen). Zusätzlich beeinflussten sicherlich auch das Bühnenbild und andere visuelle Elemente wie sie in den Regieanweisungen und (auch) in einigen gesungenen Worten nahegelegt werden - die Reaktion des Publikums.

Als eindeutig exotisierende Momente und Details in Metastasios Libretto lassen sich nennen:

1) In der Mitte des I. Aktes kommt Cleofide in Alessandros Lager, und zwar der Regieanweisung gemäß „mit [...] vielen Indern, verschiedene Geschenke tragend“ von (in Cleofides Worten zu Alessandro) den „Klippen“ und der „weiten östlichen Meeresküste“ (I 13). ${ }^{13}$ Die Regieanweisung im Libretto für die aufwendige Premiere von Hasses Oper in Dresden präzisiert sogar noch diese einheimischen Gaben: „Tiger, Lözen und Körbe mit Gold und Perlen" ${ }^{14}$

2) An einer früheren Stelle in Metastasios Libretto nehmen zwei Inder Poros Schwester Erissena gefangen und bringen sie in Ketten zum griechischen Kom-

11 Thomas Betzwieser: Exotismus und ,Türkenoper in der französischen Musik des Ancien Régime: Studien zu einem ästhetischen Phänomen (Neue Heidelberger Studien zur Musikwissenschaft; 21). Laaber 1993, 16-17.

12 Besonders wenn man sich die obligatorischen italienischen Endungen wegdenkt, wird aus ,Gandarte' wieder ,Gandharta', wie in einer deutschsprachigen Aufnahme bei den Händelfestspielen in Halle von 1959: Händel: Poros [sic], dirigiert von Horst-Tanu Margraf. Edel CD 0093742BC. (Neuveröffentlichung 1998).

13 Der Einfachheit halber wird hier - sofern nicht anders angegeben - die Nummerierung der Akte und Szenen aus Metastasios Alessandro-Libretto von 1729 verwendet, auch wenn auf die Vertonungen von Händel und Hasse Bezug genommen wird; Auslassungen und Einfügungen durch die jeweiligen Bearbeiter des Librettos und die Komponisten dieser Opern führten zu einer etwas anderen Nummerierung der Szenen. Das Libretto von 1729 (veröffentlicht 1730) kann auf der Paduaner Webseite für Metastasios Libretti eingesehen werden: http://www.progettometastasio.it, 29. März 2016. In jeder Produktion oder Aufnahme können sich die Akt- und Szeneneinteilungen ändern, abhängig davon, wie viele Pausen es gibt, welche Nummern ausgelassen beziehungsweise gekürzt werden et cetera.

14 Hasse: Cleofide I 14. Das Libretto für diese Oper wurde in der ersten Auflage der hervorragenden CD unter der musikalischen Leitung von William Christie (Capriccio 10 193/96) als Faksimile nachgedruckt, aber nicht in der Neuauflage dieser Aufnahme im Jahr 2011 (Capriccio 7080). Es wurde auch in der kritischen Ausgabe, herausgegeben von Zenon Mojzysz, nachgedruckt, aber nicht in dem auf der kritischen Ausgabe basierenden Klavierauszug. Meine Übersetzungen basieren frei auf denen aus dem Beiheft der Aufnahme von Liesl B. Sayre und der kritischen Ausgabe (von Liesl B. Sayre [et al.] für das Englische und von Zenon Mojzysz [et al.] für das Deutsche). Händels Poro re dell'Indie ist als eine ebenso bemerkenswerte CD-Aufnahme zugänglich, unter der musikalischen Leitung von Fabio Biondi (Opus 111 OPS 30-113/115). 
mandanten, um sich bei ihm einzuschmeicheln (I 3). Alessandro betrachtet dies als einen abscheulichen Akt. Er nennt die beiden Inder verräterische Schurken („Indegni!“) und erklärt Erissena, dass er - im Gegensatz zu „anderen Feinden", womit er wahrscheinlich die Perser oder Skythen meint - Schönheit „respektiert“ und keineswegs wünscht Erissena zu „schänden“ („oltraggiarti“). Alessandro befreit die indische Prinzessin und schickt die beiden „Verräter" in Ketten zurück zu Poro, damit er sie bestrafen kann. Damit soll vermutlich angedeutet werden, dass das frauenfeindliche Verhalten und der dreiste Egoismus der beiden Verräter typisch für Länder sei, die weit entfernt von Griechenland liegen, der Heimat der aufgeklärten Gesinnung und moralischen Standhaftigkeit. So zeigt der westliche Held des Librettos die kulturellen Eigenschaften indischer Männer - und, allgemeiner: der männlichen Bewohner weit östlich von Europa gelegener Gegenden - in einem unvorteilhaften Licht zum Vergnügen des lesenden und hörenden Publikums.

3) Ein zentrales Element der letzten Szene von Metastasios Libretto ist eine kulturelle und religiöse Sitte, die eindeutig indisch ist, nämlich die hinduistische Tradition der Witwenverbrennung (Sati), bei der sich eine Witwe freiwillig verbrennen lässt - normalerweise auf dem Scheiterhaufen für ihren toten Ehemann. Dieser Brauch wird in vielen europäischen Schriften des 18. Jahrhunderts erwähnt. ${ }^{15}$ Gegen Ende von Metastasios Libretto Alessandro nell'Indie (III 12) ist Cleofide verzweifelt, nachdem sie irrtümlich informiert wurde, ihr geliebter Poro habe sich in den Hydaspes gestürzt, um der Gefangennahme durch die Griechen zu entgehen. Cleofide bereitet sich vor, einen Scheiterhaufen zu besteigen, um der „Sitte“ zu gehorchen, die durch ein „uraltes Gesetz“ vorgeschrieben ist. Sie erwähnt, dass der Rauch - den das Publikum in diesem Augenblick vermutlich zu sehen beginnt - mit Gewürzen parfümiert ist. Damit findet sich im gesungenen Text ein weiteres exotisches Element: Gewürze waren zu Händels Zeit eines der wichtigsten Güter im Handel mit dem Osten. Alessandro, der konsterniert ist, Cleofide am Fuß des Scheiterhaufens zu sehen, spricht die (damals wie heute) archetypisch westliche Reaktion aus: Die Forderung, dass eine Witwe sich selbst verbrennen soll, sei „ein unmenschliches Gesetz, das man aufhalten muss, [und] das ich zu vernichten wissen werde“ („legge inumana, che a bisogno di freno, che distrugger saprò").

4) Als Cleofide dabei ist, das Ritual der Witwenverbrennung durchzuführen, versteckt sich Poro in einem nahe gelegenen heidnischen Tempel, um Alessandro und Cleofide zu erstechen. Er glaubt nämlich irrtümlich, dass Cleofide zarte Gefühle für den griechischen Heerführer hegt. Als er sie belauscht und beobachtet, erkennt er die wahre Hingabe seiner Geliebten und tritt rechtzeitig

15 Zum Beispiel berichtet Gottfried Taubert in Kompendium zur Tanzkunst von 1717, dass Frauen, welche die Witwenverbrennung verweigerten, zu Prostituierten und lasziven Tänzerinnen würden (Gottfried Taubert: Rechtschaffener Tantzmeister, oder, Gründliche Erklärung der frantzösischen Tantz-kunst. Leipzig 1717, 59-60). 
hervor. Damit rettet er sie vor dem Scheiterhaufen. Indem Poro rechtzeitig handelt, ergibt sich ein weiterer Vorteil (für den Librettisten sowie für die westlichen Prinzipien der Toleranz und Duldsamkeit), nämlich dass Alessandro, der edelgesinnte imperiale Eroberer, sich nicht mehr mit Gewalt gegen die lokale Sitte stellen muss. ${ }^{16}$

5) Wir wissen nicht, ob bei der eindrucksvollen Premiere von Hasses Cleofide in Dresden tatsächlich echte Löwen und Tiger - oder Tänzer, die als solche verkleidet waren - auf die Bühne kamen oder ob das Opernpublikum sich diese anhand der gedruckten Regieanweisungen in ihren Libretti vorstellen musste. Wir wissen aber, dass es in dieser Produktion mehrere andere Elemente gab, mit denen der zeitliche Rahmen und die ethnischen Aspekte der Geschichte verdeutlicht werden sollten. Nach einem zeitgenössischen Bericht nahmen 54 Soldaten aus König Augusts Armee in der Schlachtenszene teil (II 5). Sie haben „das Ihrige bei der Bataille in der Opera mit gutem Fleiß und Geschicklichkeit verrichtet, wie sie denn mit Schildern auff die alte Griechische und Indianische Art zu fechten einige Zeit vorher exerciret worden" ${ }^{17}$ Die Aufführung wartet auch mit drei unabhängigen balli auf, einer nach jedem Akt der Oper. ${ }^{18} \mathrm{Im}$ ersten dieser balli traten „marinari“ (Seeleute) auf - vielleicht die Tänzer oder Schauspieler, die Cleofide und ihre Geschenke einige Minuten zuvor mit dem Schiff zu Alessandro gebracht hatten. Zum ballo nach dem II. Akt gehörten „Indiani“ (weitere Details sind nicht überliefert) und der nach dem III. und letzten Akt bestand aus einem Tanz von lokalen „Bacc[h]anti“; damit waren im Kontext dieser Oper nicht weibliche Anhängerinnen des Dionysus beziehungsweise Bacchus gemeint, sondern einer indischen Gottheit der irdischen Liebe, die vermutlich als ungefähr äquivalent zu diesen westlichen Göttern angesehen wurde (vielleicht Kāmadeva, auch bekannt als Māra). ${ }^{19}$

16 Metastasio (1730), III 13. Cleofide hatte schon in einer früheren Szene der Oper darüber nachgedacht, sich in den Hydaspes zu werfen. Dies entspricht nicht gerade dem Verhalten westlicher Königinnen in Opern dieser Zeit, insbesondere nicht solchen, die als im Grunde bewundernswert dargestellt werden. Poro und Gandarte drohen in Metastasios AlessandroLibretto ebenfalls mit Selbstmord im Verlauf der Oper. In Händels Giulio Cesare ist Cornelia, die Witwe des ermordeten römischen Generals Pompeo, eine bemerkenswerte Ausnahme; ihre Hoffnungslosigkeit war möglicherweise wenigstens verständlich zu dieser Zeit, da ihr schreckliches Entsetzen darüber gezeigt wird, in den Harem von Tolomeo gezwungen zu werden.

17 Aus einem quasi-offiziellen Bericht über den Tag in Curiosa Saxonica 75, 1731, 271, zitiert in: Zenon Mojzysz: Cleofide, „Dramma per musica“ von Johann Adolf Hasse. Untersuchung der Entstehungsgeschichte (Hasse-Studien / Sonderreihe; 2). Stuttgart 2011, 54.

18 Die Musik und Choreografie dieser drei balli ist leider verloren. Jedoch werden die balli in der Originalausgabe des Librettos an prominenter Stelle erwähnt. Im Klavierauszug und in der Neuauflage der Capriccio-CD werden die balli nicht erwähnt.

19 Die letzte Szene der Oper mit dem Scheiterhaufen findet vor einem „Tempel des Bacchus“ statt. Händels Oper tilgt unter anderem den Chor aus den III. Akt und den ballo für die Bacchantinnen (in diesem Fall die Tempelpriesterinnen) und vereinfacht im Verlauf der Oper einige der szenischen Effekte, jedenfalls nach den Regieanweisungen aus dem veröf- 
Alle diese Details bestätigen Reinhard Strohms Annahme, dass Hasses Cleofide in ihrer vollständigen Fassung auf der Dresdener Bühne 1731 „ein relativ frühes Beispiel für echte Exotik und Chinoiserie im Musiktheater" war. ${ }^{20}$

\section{Metastasios Raja als Antiimperialist und schlechter Anfübrer}

Die Auseinandersetzung des Libretto mit der Frage nach europäischer Identität und nicht-europäischer Alterität zeigt sich auch in einem anderen hervorstechenden Merkmal: dem wiederholten Anprangern des griechischen Imperialismus. Nachdem Cleofide Alessandro ihre Gaben präsentiert, erinnert sie ihn an die Städte und Felder, die er in ihrem Land zerstört habe, und das „extreme Leid“, das dies verursacht habe. Was Poro anbelangt, so ist er noch immer Herrscher über große Gebiete Indiens, obwohl er emotionale Schwächen zeigt. Er erhält ebenfalls die Gelegenheit, seinem Unmut über die Griechen freien Lauf zu lassen und zwar in einer Weise, die auf jüngere Beispiele westlicher Gier anzuspielen scheint. Mit zunehmender Wut klagt er den griechischen Eroberer an, und im Verlauf der Tirade wechselt seine Anrede aus der respektvollen dritten Person in die beschuldigende zweite Person: „Welches Ziel treibt denn Alessandro in die Länder der Aurora, ihren Frieden zu zerstören? [...] Nun hast du schon zum Tribut die Welt überall verpflichtet. Doch die ganze Welt ist deinem Durst zu wenig?“ (I 2). Poros General Gandarte lässt ähnliche Vorwürfe verlauten, als er, verkleidet als Poro, Alessandro gegenübertritt, genauso wie Alessandros eigener General Timagene in einem Monolog. ${ }^{21}$ Diese wiederholten Anklagen sind ein Echo der im 17. und 18. Jahrhundert oft geführten Debatten über die moralische Bewertung der zeitgenössischen imperialistischen Unternehmungen der Spanier und anderer europäischer Mächte. ${ }^{22}$ Poros lange und beindruckende Rede, die oben in Auszügen zitiert wurde, wurde fast komplett von Hasses Librettist übernommen. ${ }^{23}$ Händels Librettist hingegen kürzte

fentlichten Libretto zu schließen. Vgl. Winton Dean: Handel's Operas, 1726-41. Woodbridge 2006, 169-173.

20 Reinhard Strohm: Hasses Oper ,Cleofide‘ und ihre Vorgeschichte. In dem Beiheft zur ersten CD-Veröffentlichung der Aufnahme, die oben bereits angeführt wurde, 13-21, hier 13. Strohm meint mit „echt“ vermutlich den Versuch, zumindest ungefähr korrekt zu sein; er verwendet den Begriff, Chinoiserie' anscheinend recht frei.

21 Gandarte: „Du willst, dass wir alle sterben“ (Libretto von 1729, II 13); im Libretto für Cleofide (bearbeitet von Boccardi) folgt darauf: „Es gefiele dir, wenn der ganze Indus rot von Blut wäre“. Timagene erklärt seine Absicht, die „unterdrückte Welt" vom „Joch“ Alexanders zu befreien (Alessandro-Libretto von 1729, II 11).

22 Vgl. Locke: Music and the Exotic (Anm. 2), 76-77, 90-91, 230-232. Der Hörer vertraut Timagenes Äußerungen nicht ganz, weil Timagene, wie es im Libretto steht, Alessandros „geheimer Feind" ist. Timagene ist der Sohn von Cleitos, den Alexander der Große betrunken in einem Kampf getötet hat; und außerdem hat Alessandro gerade Timagenes Pläne vereitelt, Zugang zu Poros Schwester Erissena zu erlangen. Aber wir erkennen in diesen Worten auch eine echte westliche Sympathie für eroberte Völker.

23 Ausgelassen wurden einige spezifische Aussagen über Afrika und Asien. 
sie drastisch, vielleicht aus Rücksicht auf die zeitgenössischen britischen Aktivitäten in Indien. ${ }^{24}$

So bewundernswert Raja Poros Ziele sein mögen, ihm fehlt Alessandros emotionale Stabilität und dessen Sinn für Prioritäten. ${ }^{25} \mathrm{Im}$ Verlauf der Oper verdächtigt Poro Cleofide immer wieder der Untreue. ${ }^{26}$ Als er hört, dass Cleofide auf dem Weg sei, sich mit Alessandro zu treffen (I 9), befürchtet er, sie könnte nun dem westlichen Eroberer ihre Zuneigung schenken, und will sie sofort verfolgen. Sein General Gandarte erinnert ihn unverzüglich daran, dass er stattdessen den Kampf gegen die griechische Invasionsarmee anführen sollte. ${ }^{27}$ In einer anderen Szene (II 7) eine der wenigen, in denen Poro nicht von der Eifersucht beherrscht ist - offenbart er eine weitere problematische Eigenschaft: Impulsivität. Nachdem er - als Asbite, also als ein Mitglied des indischen Hofs, verkleidet - Alessandros Lager am Flussufer erreicht hat, enthüllt er aus Stolz seine wahre Identität. ${ }^{28}$ Cleofide unterbricht ihn, kurz bevor er seinen wirklichen Namen preisgibt.

Im Gegensatz zu dieser Unbeständigkeit demonstriert Alessandro - in jeder Hinsicht das Beste, was der Westen zu bieten hat - bei vielen Gelegenheiten Milde und Selbstverleugnung. Er zeigt sogar Reue über das Leid, das er Indien gebracht hat, und beharrt darauf, dass er die Frauen in jedem eroberten Land respektiere. Erneut bestätigt sich diese Selbstbeherrschung am Ende der Oper. Obwohl Alessandro von Cleofide bezaubert ist, steht er der Wiedervereinigung mit ihrem langjährigen Geliebten Poro nicht im Wege. Er setzt beide auch wieder als Herrscher über ihre benachbarten Reiche ein, wenn auch in einer untergeordneten Position: als Monarchen, die einem imperialen Griechenland tributpflichtig sind.

Händel hatte fünf Jahre zuvor bereits eine Oper mit dem einfachen Namen Alessandro komponiert, allerdings mit einem ganz anderen Libretto, das den griechischen Eroberer in ein weniger vorteilhaftes Licht rückt. Zum einen erklärt er sich zum Gott, nachdem er eine indische Stadt (Oxidraca, ein erfundener Name) erobert hat - für diesen Akt des Größenwahns wird er prompt von dem aufrechten

24 Reinhard Strohm: Metastasio's ,Alessandro nell'Indie ${ }^{6}$ and Its Earliest Settings. In: Reinhard Strohm: Essays on Handel and Italian Opera. Cambridge 1985, 232-248, hier 239. Einige von Händels Freunden und Investoren seines Opernensembles waren auch am Überseehandel beteiligt. Vgl. Ellen T. Harris: With Eyes on the East and Ears in the West: Handel's Orientalist Operas. In: Journal of Interdisciplinary History 36: 3, 2006, 419-443, besonders 432-434. Harris: George Frideric Handel (Anm. 5), 76-113.

Dean kennzeichnet seinen Charakter treffend als „instabil und frenetisch, von Liebe und Eifersucht besessen“ (Dean: Handel's Operas [Anm. 20], 180).

26 Vgl. Alvaro in Rameaus Les Indes galantes: Locke: Music and the Exotic (Anm. 2), 236.

27 In Hasses Libretto (in der Bearbeitung von Boccardi) heißt es „als Schwächling erscheinen“ („debole comparir"). Die Worte, die Metastasio Gandarte an dieser Stelle in den Mund legt, scheinen Poro gegenüber respektvoller zu sein: „[deine] großen Vorhaben zu zerstören“" (,scomporre i gran dessigni“).

Bei Händel: II 3; bei Hasse: II 7. 
General Clito kritisiert. ${ }^{29}$ Zum anderen fühlt dieser Alexander sich nicht nur zu einer östlichen Prinzessin, sondern sogar zu zweien hingezogen: Rossane, welche die Griechen als Gefangene aus Persien mitgebracht haben, und Lisaura, eine Skythin und damit in Alessandros Sicht eine „Barbarin““ ${ }^{30}$ Ein Teil der Kunst einer Oper besteht darin, bekannte Elemente neu zu arrangieren. Vielleicht kamen manche Londoner Opernbesucher, die beide Händel-Opern sahen, sogar zu dem Schluss, dass Alexander der Große, nachdem er seine Lehren aus der Oper Alessandro im Jahre 1726 gezogen hat, sich fünf Opernsaisons später in seine kulturelle Rolle als bewundernswerte Heldenfigur gefügt hatte.

Metastasios Entscheidung, Alexander in Alessandro nell'Indie als affektkontrolliert zu präsentieren, hat den Vorteil, dass die Rolle des vernarrten Geliebten von einem anderen Mann gespielt werden konnte, und zwar nicht von einem anderen griechischen (oder römischen) Krieger, wie es in Opern aus dem frühen 18. Jahrhundert sonst oft der Fall war, sondern von einem indischen Raja. Indem er einem östlichen Mann moralische Schwäche zuschreibt, stützt sich der Librettist auf gängige Klischees über nicht-europäische Männer. Die Verbindung von fremder Ethnizität und Charakterschwäche entsprach kontrastiv dem europäischen Selbstbild und unterstützte mittelbar die imperialistischen Eroberungen und Handelsabkommen, die oft militärisch durchgesetzt wurden. ${ }^{31}$ Wurde ein westlicher Mann vom Liebesverlangen übermannt - zum Beispiel Grimoaldo, der Herzog von Benevento, in Händels Rodelinda -, konnte er dennoch als potenziell guter Herrscher gedeutet werden, der lediglich von einem zynischen und ehrgeizigen Berater beeinflusst wird (Garibaldo). Dagegen erscheinen Poros wiederkehrende Habgier, Eifersucht und die Tatsache, dass er in der Schlussszene der Oper Cleofide und Alessandro auflauert, um sie zu ermorden, als Ausdruck einer inhärenten Unterlegenheit, ganz gleich, ob diese nun ethnisch, rassisch oder kulturell interpretiert wurde.

\section{Verschiedene musikalische Arrangements und ibre Wirkung}

Ein Komponist, der Metastasios Libretto vertont, kann durch die Musik die Unterschiede im Verhalten der Repräsentanten des Westens und des Ostens entweder hervorheben oder herunterspielen. Für Autoren, die sich mit Händels und Hasses

$29 \mathrm{Zu}$ Clito in Alessandro (einer Figur, die auf der historischen Gestalt Cleitos basiert), vgl. Regina Compton: How to Enrage Alexander, or Towards an Understanding of Handel's Recitativo semplice and Theatrical Gesture. In: Händel-Jabrbuch, 62, 2016, 33-51.

30 Rossane ist in dieser Oper zweifellos das Äquivalent zur historischen Roxana, eine bekannte baktrische Frau, die Alexander 327 heiratete. Aber einige ihrer Eigenschaften scheinen denen einer Figur mit einem ähnlichen Namen, jedoch aus einer sehr viel jüngeren Zeit nachempfunden zu sein (siehe Anm. 41).

31 Zur Einstellung zu den europäischen Überseeeroberungen und den militärisch erzwungenen Handelsabkommen im frühen 18. Jahrhundert, besonders in Indien, Nord- und Südamerika und gegenüber dem Osmanischen Reich, vgl. Locke: Music and the Exotic (Anm. 2), 56-74, 75-100, 204-207 und 264-269. 
Alessandro nell'Indie-Opern befasst haben, ist diese Frage meist nicht von zentraler Bedeutung. Sie beschäftigen sich stattdessen mit anderen (ebenfalls berechtigten) Fragen, etwa wie sich diese Opern zu den Konventionen und Themen der barocken Opera seria verhalten. ${ }^{32}$ So argumentiert Reinhard Strohm zum Beispiel, dass Händels Poro der Intention von Metastasios Werk treu bleibe, indem er „den Kampf zwischen den Persönlichkeiten und Nationen [die im Zentrum früherer Versionen, etwa in Racines Stück, standen] auf die geistige Ebene des individuellen Gewissens versetzt" ${ }^{\text {“ }}{ }^{33}$ Sven Hansell betont einen ähnlichen Gesichtspunkt: Metastasios Alessandro-Libretto lenke unsere Aufmerksamkeit von den Fragen geopolitischer Konflikte auf die verschiedenen amourösen Verwicklungen und Missverständnisse der einzelnen Figuren. In Hasses Vertonung Cleofide intensiviere der „kokettierende“ Ton der Musik diese Verlagerung. ${ }^{34}$

Stimmung und Stil der verschiedenen musikalischen Stücke der beiden Alessandro/Poro/Cleofide-Opern variieren stark. Auf zwei Aspekte dieser vielfältigen Stimmungen und Stile möchte ich kurz eingehen, bevor ich einige Arien und Rezitative aus beiden Opern untersuchen werde.

1) Sowohl Händel als auch Hasse komponierten einen etwas merkwürdigen, vielleicht sogar exotisch klingenden orchestralen Marsch für den Moment, als Cleofide mit ihrem Gefolge von Geschenke tragenden Indern auftritt. In Händels Marsch (Poro: I 9) findet sich ein wiederholter, schwerfälliger Rhythmus aus drei langen Tönen, vielleicht ein Verweis auf die Figur des „exotischen Marsches", die - wie Miriam Whaples bemerkt hat - in der berühmten türkischen Szene von Molières und Lullys Le bourgeois gentilhomme eingeführt wurde und die für mehr als ein Jahrhundert einen Nachhall in Bühnenarbeiten von Rameau, Gluck, Dalayrac und Mozart fand. ${ }^{35}$ Hasses Marsch (Cleofide: I 14) besteht aus einer lebendigeren und punktierten, aber genauso sturen und wenig anmutigen Figur. Diese unerwarteten, sogar unbeholfenen stilistischen Feinhei-

32 Dean: Handel's Operas (Anm. 19), 169-93. Frederick L. Millner: The Operas of Johann Adolf Hasse (Studies in Musicology; 2). London 1979, 103. Sven Hansell: Cleofide. In: Grove Music Online, http://www.oxfordmusiconline.com/subscriber/article/grove/music/O901081, 29. März 2016. Strohm: Hasses Oper ,Cleofide' (Anm. 21), 17. Roland Dieter SchmidtHensel: ,La musica è del Signor Hasse detto il Sassone...' Johann Adolf Hasses, Opere serie‘ der Jabre 1730 bis 1745: Quellen, Fassungen, Auffübrungen (Abhandlungen zur Musikgeschichte; 19.1-2). Göttingen 2009, Bd. 1: 210, 214, 216, 218, 226, 302; Bd. 2: 153-178. Dass die verschiedenen Verwicklungen in der Handlung in Dresden sehr wohl als Andeutungen auf bestimmte Personen am Hof verstanden worden sein könnten, argumentiert Strohm: Hasses Oper ,Cleofide' (Anm. 21), 31. Diese Interpretation wird hinterfragt von Panja Mücke: Johann Adolf Hasses Dresdner Opern im Kontext der Hofkultur. Laaber 2003, 88. Strohms Sichtweise wird unhinterfragt übernommen von T.C.W. Blanning: The Culture of Power and the Power of Culture: Old Regime Europe, 1660-1789. Oxford 2002, 64-67.

33 Strohm: Metastasio's ,Alessandro nell'Indie' (Anm. 24), 232, 236 (Zitat), 241, 245.

34 Hansell: Cleofide (Anm. 32).

35 Locke: Music and the Exotic (Anm. 2), 227-228, 313-315. Die Harmonie in Händels Marsch neigt auch dazu, zu lang auf der Tonika zu verweilen, um sich dann plötzlich in andere Tonarten zu stürzen. 
ten könnten auch in der Art und Weise inszeniert worden sein, wie die Inder auf der Bühne marschierten, gestikulierten oder kostümiert waren; ${ }^{36}$ vielleicht die einzigen Momente in beiden Werken, die sich dem Paradigma des exotischen Stils zurechnen lassen.

2) Auch wenn in einer Oper eine oder mehrere exotische Figuren auftreten, sollten wir vorsichtig sein, die üblichen Generalisierungen im Hinblick auf Opern aus dem frühen 18. Jahrhundert zu übernehmen. Der Hasse-Fachmann Zenon Mojzysz meint etwa, die Figuren der Cleofide seien "relativ einfach konzipiert“ und man könne „sogar jedem Charakter einen einzigen beherrschenden Affekt zuordnen". Cleofides Antriebskraft zum Beispiel sei die Liebe. ${ }^{37}$ Auch wenn dies zutreffen mag, so ist doch zugleich festzuhalten, dass Cleofide die Tiefe ebendieses einen Gefühls, ihrer Liebe zu Poro, auf ganz verschiedene Weisen auszudrücken vermag, die wiederum unterschiedlichen Charaktereigenschaften entsprechen. Dies macht sie zu einem gewissen Grad ambivalent und unberechenbar. Cleofide folgt einer durchdachten Strategie, um Alessandro zu Zugeständnissen zu überreden; sie zeigt Selbstbewusstsein und berechtigte Wut, nachdem Poro ihr unterstellt, sie sei nun Alessandro zugeneigt. Sie gibt sich verständlicher Verzweiflung hin, nachdem sie das falsche Gerücht von Poros Tod hört. Vielleicht am eindrücklichsten ist die Szene (I 13), in der sie durch einige schnelle taktische Entscheidungen fast ihr Ziel erreicht, Alessandros Begehren für sich zu wecken. So droht sie zum Beispiel abzureisen, als er ihre Geschenke zurückweist, denn sie könnte nirgends bleiben, wo ihre Gaben nicht willkommen wären. Dadurch ist Alessandro gezwungen, sie darum zu bitten, bei ihm zu bleiben. Als er im Versuch, seine Liebe zu ihr zu dämpfen, ihren Blick nicht erwidert, täuscht sie vor zu weinen. Cleofide deutet auch mehrfach an, sie sei von Alessandro bezaubert - sie preist laut „seine milden Züge, seinen achtungsvollen Blick und seine höfliche Rede“ - und erklärt schließlich, als ihr wahrer Geliebter Poro verkleidet als Asbite erscheint: „Für Alessandro allein empfinde ich Liebe und bekenne [...] Gefühle, die ich mit viel Mühe, o Herr, bisher verschwiegen habe.“ Während dieses langen und sehr manipulativen „Angriffs“ auf Alessandro - er verwendet diesen Begriff ad spectatores - hält der griechische Eroberer mehrmals inne, um seine Fassung wiederzugewinnen und singt schließlich eine Abgangsarie, in der er sanft aber bestimmt erklärt, dass er ihr Verteidiger und Freund sein wolle, ihr aber sein Herz nicht schenken könne. ${ }^{38}$

Verführerisches und heuchlerisches Verhalten wurde in Opern des 18. Jahrhunderts oft westlichen Frauenfiguren zugeschrieben (zum Beispiel Poppea in Händels Agrippina und Antigona in seiner Oper Admeto). Nichtsdestoweniger könnte es sein,

$36 \mathrm{Zu}$ den absichtlich ungeschickten, unkoordinierten oder schwerfälligen Bewegungen von „Indern“ aus verschiedenen Kontinenten im Ballett des Barock vgl.: Locke: Music and the Exotic (Anm. 2), 12-13, 83-84 (Abb. 4.5 und 4.6), 139-142, 146, 149.

37 In der Einführung zum Klavierauszug: vii-viii.

38 Er flüstert sich selbst Sätze zu, wie „Alma[,] costanza“ (,Sei ruhig mein Herz!'). 
dass Metastasio in diesem Libretto die verführerischen und heuchlerischen Worte und Taten Cleofide vor allem deshalb zugeschrieben hat, weil sie dem Osten und nicht Europa entstammt. ${ }^{39}$ Hinter Cleofide stehen - in der Vorstellung gebildeter Personen des frühen 18. Jahrhunderts - die weitverbreiteten Bilder von mächtigen morgenländischen Frauen, zu denen nicht nur die große Kleopatra von Ägypten gehört, sondern auch muslimische Frauen wie Roxana (oder Roxolana), die, obwohl sie in der heutigen Ostukraine geboren wurde, Mitte des 17. Jahrhunderts in den Harem des osmanischen Sultans Suleimans I. kam. ${ }^{40}$

\section{Die Arien als Charakterisierungsmittel}

Cleofides Versuch, Alessandros Gefühle zu beeinflussen, wird nur im Rezitativ vorgetragen. Die wechselnden Effekte, die ich beschrieben habe, hängen stark von den Fähigkeiten und der Sensibilität der jeweiligen Sängerin und den Mitgliedern der Continuo-Gruppe ab. Aber auch die Arien für Cleofide und Poro deuten oft exotische Elemente an, die direkter in den Noten der Partitur verankert sind und deshalb jede wenigstens halbwegs kompetente Darbietung beeinflussen mussten. Marita P. McClymonds hat darauf hingewiesen, dass es sich bei einer von Poros Arien, nämlich „Generoso risvegliati, o core“ (II 4), vielleicht um eine Frühform des komischen türkischen Stils handeln könnte. Hasse hatte diese Arie aus einer seiner früheren Opern übernommen, Gerone, tiranno di Siracusa (Neapel, 1727). ${ }^{41}$ Wie die oben erwähnten Märsche könnte diese Ausnahme vielleicht als frühes Beispiel des ,exotischen Stils' betrachtet werden. Freilich bleibt fraglich, ob das

39 Atalanta aus Händels Serse teilt einige dieser Eigenschaften; sie ist wie die anderen Figuren jener Oper eine Perserin.

40 Über Roxana behauptete man, es sei ihr mit weiblichen Waffen gelungen, Suleimans einzige Frau zu werden, und sie habe es durch Klugheit und Hinterlist vermocht, einen Sohn, den er mit einer früheren Frau hatte, ermorden zu lassen, so dass ihr eigener Sohn der Nachfolger von Suleiman werden konnte. Vgl. Porträts und Informationen in Locke: Music and the Exotic (Anm. 2), 64-74. Die listige und willensstarke Rossane in Händels Alessandro - sie verlangt an einer Stelle, freigelassen zu werden - spiegelt Eigenschaften, die im frühen 18. Jahrhundert meist der historischen Roxana/Roxolana aus dem 16. Jahrhundert zugeschrieben wurden.

41 Marita Petzoldt McClymonds: Style as Sign in Some opere serie of Hasse and Jommelli. In: Reinhard Wiesend (Hg.): Johann Adolf Hasse in seiner Zeit: Bericht viber das Symposium vom 23. bis 26. März 1999 in Hamburg (Hasse-Studien / Sonderreihe; 1). Stuttgart 2006, 179-192, hier 181. McClymonds hat vielleicht gespürt (obwohl sie es nicht erwähnt), dass es eine spezifische musikalische Ähnlichkeit zwischen dieser Arie und einer anderen gibt, die von einem türkischen Protagonisten in einer Oper gesungen wird, die fünfzig Jahre später komponiert wurde: Osmins „O wie will ich triumphieren“, aus dem III. Akt von Mozarts Die Entführung aus dem Serail (1781). Der zweite Takt von Hasses Eröffnungsritornell donnert fünf Achtelnoten heraus und dann zwei Viertelnoten; das zweite Auftreten dieses rhythmischen Motivs endet auf der Tonstufe 3. Osmin singt fast das gleiche rhythmische Motiv („wie will ich triumphieren“, das mit sechs wiederholten Noten beginnt) und endet jeweils mit der Tonstufe 3. Beide Komponisten begleiten das rhythmische Motiv mit Oktavsprüngen im Orgelpunkt. 
Publikum bereits 1731 diese ungewöhnlichen stilistischen Aspekte in dieser Arie als eindeutig nicht-europäisch erkannte.

Typisch für diese beiden Opern ist es eher, dass Händel und Hasse das Exotische inszenieren, obne auf ,fremde' (oder eben sonderbare) stilistische Elemente zurückzugreifen. Die bekannteste Arie aus Hasses Cleofide ist „Digli ch'io son fedele“, gesungen von der Titelfigur. ${ }^{42}$ Cleofide richtet diese Arie an Alessandros General Timagene, während Poro als Asbite verkleidet anwesend ist. Sie versichert Poro ein weiteres Mal, dass sie ihn liebe und dass er seine Ungeduld zügeln solle: „Sag ihm [Poro], ich bleibe ihm treu [...] und er solle noch nicht verzweifeln.“ Der Text zeigt erneut, dass die indische Königin in der Lage ist, ihre eigenen Ziele zu verfolgen, ohne den Verdacht oder Widerstand der anderen Anwesenden zu erregen. Zugleich erreicht die Musik im ausgewogenen Zusammenspiel von Melodik und kunstvoller dekorativer Wirkung eine Art parallele Virtuosität, die uns durch die metaphorische Sprache kunstvollen Komponierens und grandiosen Gesangs - daran erinnert, warum sowohl Alessandro als auch Poro sich von dieser bemerkenswerten Frau angezogen fühlen. ${ }^{43}$

Eine frühere Szene zeigt noch anschaulicher, wie auch ohne exotische Stilmittel eine exotische Charakterisierung erreicht werden kann. In Metastasios Libretto (I 9) sehen wir erneut Raja Poros Schwierigkeiten, seine Gefühle zu kontrollieren. Gandarte hat ihn gerade angefleht, sich nicht länger in Cleofides mögliche Untreue hineinzusteigern. Poro gibt zu, dass er seinen militärischen und patriotischen Pflichten nicht nachgekommen ist (,Du sprichst die Wahrheit, Freund, ich weiß es"), beginnt aber dann mit einer Arie, in der er versucht, sein feiges Verhalten zu entschuldigen. Der Text der Arie („Se possono tanto | Due luci vezzose“) argumentiert, niemand, der nicht selbst dem Zauber zweier schöner Augen verfallen ist, könne verstehen, welche Qualen eine verliebte Person erleide. Metastasios Verse legen einem Komponisten alle möglichen Vertonungen nahe. Händels Musik in Poro betont das sinnliche Verlangen und den selbstmitleidigen Charakter des indischen Herrschers, indem er Poros Arie eine schleppende, zögerliche Qualität gibt, von Reinhard Strohm als „äußerst stilisierter Menuettrhythmus“ bezeichnet. Die zarte, unheroische, vielleicht weibliche Qualität der Musik wird durch Händels Entscheidung unterstützt, die Orchestrierung auf die Streicher zu beschränken. Sie zeigt sich besonders deutlich im zarten Murmeln der Geigen senza Cembalo, das zuerst in den Takten 5 bis 8 zu hören ist. ${ }^{44}$

42 Diese Arie, mit einem ergänzenden Kommentar versehen, findet sich in vielen Ausgaben der Norton Anthology of Western Music, die in den USA im Bachelorstudium der Musikgeschichte sehr weit verbreitet ist.

43 Für die Premiere wurde die Rolle geschrieben für Hasses Frau, die berühmte Faustina Bordoni, und auch von ihr verkörpert.

44 Im Gegensatz dazu vertritt Strohm die Ansicht, Händels Vertonung wolle durch ihre Ernsthaftigkeit aufdecken, dass Poro „von der Tatsache gequält ist, dass er so vollständig von Eifersucht beherrscht ist“ (Strohm: Metastasio's ,Alessandro nell'Indie' [Anm. 24], 241). Vgl. Dean: Handel's Operas (Anm. 19), 176-177. 
Hasses Cleofide geht mit diesen Versen anders um. Das Ritornell fügt Blasinstrumente zur Basis der Streicher und des Continuo hinzu, um eine größere Kraft zu entfalten. Poros Zeilen weisen „aggressive Rhythmen“ und „herausstechende Spitzentöne" auf, wie sie Strohm richtig beschreibt. ${ }^{45} \mathrm{Im}$ Zusammenspiel mit den Worten ergibt sich der Effekt eines fast monomanischen Zorns. Hasses Poro scheint in verdrehter Weise stolz darauf zu sein, seiner leidenschaftlichen Eifersucht ausgeliefert zu sein, anstatt das Kommando über seine Armee zu übernehmen und die westlichen Invasoren zu bekämpfen. All diese verbalen und musikalischen Merkmale müssten zumindest einige der Opernbesucher in Hasses Zeit dazu veranlasst haben, Poro als wirklich fremd und kaum bewundernswert wahrzunehmen, besonders angesichts der würdevollen Worte und der im Allgemeinen sanften und zurückhaltenden Musik in den Arien des griechischen Eroberers Alessandro. ${ }^{46}$

Händel und Hasse präsentieren Poros Charakter auf ihre je eigene Weise als ein starkes Indiz für Indiens unzulängliche soziale Werte. Ähnliche Stereotype des liebestollen östlichen Mannes findet man auch in anderen Opern Händels, wie etwa Tolomeo, deren Titelheld auf dem ägyptischen Herrscher Ptolemaios Lathyros basiert, und in Serse, angelehnt an überlieferte Geschichten über Xerxes, den großen persischen Eroberer. ${ }^{47}$ Ein noch extremeres, ekelerregendes Beispiel bietet ein

45 Strohm: Hasses Oper ,Cleofide‘ (Anm. 20), 20. Der Text von Metastasios Arie an dieser Stelle („Se possono tanto“) wurde von Hasse und/oder Boccardi mit einer Musik aus einer Wut-Arie (für eine Königin aus dem Gebiet der heutigen Türkei) untermalt, die aus einer Oper entnommen wurde, die Hasse zuvor für Neapel komponiert hatte: Attalo re di Bitinia, 1728 (Libretto von Francesco Silvani). Aber wie Strohm betont, passt die Musik ausgesprochen gut $\mathrm{zu}$ den nacheinander erfolgenden "Gesten“ von Metastasios Versen, besonders der Entwicklung vom ausdrücklichen Protest, über kurze Protestsätze des Selbstmitleids, bis zum Ende mit Koloraturgesang, der eine Art Schluchzen ausdrückt. Die letzten Absätze aus Strohms Essay mit der Diskussion dieser Arie wurden bei der Neuauflage dieser Aufnahme 2011 weggelassen, genauso wie ein Essay von Helga Lühning.

46 Poro bekommt eine weitere intensive Arie - dieses Mal mit Rache als Thema - gegen Ende von Hasses Cleofide („Dov’è? Si affretti per me la morte“); das Orchester ist besonders bewegt mit schnellen Noten im concitato-Stil der Streicher, eine Art von Musik, die Alessandro Magno in diesem Werk nie zugeschrieben wird. Hasses Oper illustriert so McClymonds Argument, dass die ernsten Opern des 18. Jahrhunderts oft die Musik mit ,hohem Stil' (tragisch, intensiv) einem schurkischen Charakter (zum Beispiel einer "starrköpfigen Hauptfigur") zuordnen und dass die Arien ,mittleren Stils" von militärischen Anführern gesungen werden, deren Benehmen eher zurückhaltend ist (McClymonds: Style as Sign (Anm. 42), 184). Als Alessandro in Cleofide eine Arie mit einem intensiven Gefühl anstimmt, geschieht dies als Reaktion auf das schlechte Verhalten einer anderen Person. Vgl. zum Beispiel die Arie „Vil trofeo d'un alma imbelle“, mit der die Szene beendet wird, in welcher der griechische Heerführer das Bitten seines Generals Timagene ablehnt, die schöne Erissena zur Sklavin zu machen. Das erinnert an Metastasios Grundprinzip, dass der Zorn eines Helden immer mit seiner sonst üblichen Selbstbeherrschung kontrastiert wird. Vgl. Francesco Cotticelli und Paologiovanni Maione: Metastasio: The Dramaturgy of Eighteenth-Century Heroic Opera. In: Anthony R. DelDonna und Pierpaolo Polzonetti (Hg.): Cambridge Companion to Eighteenth-Century Opera. Cambridge 2009, 66-84, hier 73-75, 80.

47 Dieser frühere Ptolemäer wurde zwischen 80 und 51 v. Chr. aus seinem Land vertrieben, bevor er sich mit der Hilfe Roms erneut als Herrscher über Ägypten etablieren konnte. Zu Serse, vgl. Locke: Music and the Exotic (Anm. 2), 252-253. 
anderer Tolomeo, Ptolemaios XIII. Theos Philopator, der Bruder Kleopatras, in Händels Giulio Cesare. Dieser Tolomeo zwingt der aufrechten römischen Frau Cornelia mehrmals seine Liebe auf, nachdem er ihren Ehemann Pompeo (Pompeius Magnus) in einem fehlgeleiteten Versuch, Julius Cäsar zu gefallen, hatte enthaupten lassen. ${ }^{48}$

Eine Handlung mit einem indischen Raja, der wie in Händels Poro und Hasses Cleofide äußerst besitzergreifend liebt, erschien denjenigen im Publikum besonders plausibel, denen die Mehrfrauenehe und das Ritual der Witwenverbrennung in Indien bekannt waren. Die Polygamie war aus westlicher Sicht ein Beweis dafür, dass es der indischen Frau an unabhängiger Persönlichkeit fehlte. Solche Informationsfragmente, ganz gleich wie irreführend oder stark vereinfacht sie waren, wurden durch die erotischen Erzählungen bestärkt, die das westliche Publikum des frühen 18. Jahrhunderts in den kurz zuvor veröffentlichten Übersetzungen und Adaptionen von Tausendundeiner Nacht vorfanden. Auch wenn diese Sammlung aus dem Arabischen kam und schon lange in Orten wie Bagdad und Kairo zirkulierte, war sie doch auch mit jenem großen Teil Indiens verbunden, der von der Mitte des 16. Jahrhunderts bis ins frühe 19. Jahrhundert von den muslimischen Moguln beherrscht wurde. Europäischen Lesern war dies aus Zeitungen, Geschichtsbüchern und Reiseberichten bekannt. ${ }^{49}$

Diese kurzen Einblicke in ein Libretto und zwei darauf basierende Opern erinnern uns daran, dass in sehr vielen Opern vor 1750 nicht-europäische männliche und weibliche Figuren auftraten, die heldenhaft oder schurkisch, aufrichtig oder hinterhältig waren oder diese oder andere Eigenschaften auf ganz unterschiedliche Weisen in sich vereinten. Interessanterweise ist Cleofide sowohl heldenhaft als auch hinterhältig. Besonders bemerkenswert sind männliche Protagonisten wie Poro, die sehr weit von jedem aristotelischen Verhaltensideal entfernt zu sein scheinen. ${ }^{50} \mathrm{Da}$ Opern verhaltensdidaktische Ziele verfolgten, dienten solche Darstellungen eines aufbrausenden, selbstsüchtigen Anführers auch dazu, mit dramatischen Mitteln davor zu warnen, den Pfad der Tugend zu verlassen. Aber indem diese problematischen, manchmal bösartigen und abstoßenden Figuren einem fernen Land und

48 Dieser spätere Ptolemäer führte Krieg gegen Julius Cäsar. Kleopatra, seine Schwester, schloss sich mit Cäsar zusammen, und Ptolemaios ertrank auf der Flucht beim Versuch, den Nil zu durchschwimmen. Mehr über seine Darstellung in Händels Giulio Cesare: Locke: Music and the Exotic (Anm. 2), 153-56. Locke: Alien Adventures (Anm. 1), 53-69.

49 Bestimmte Erzählungen aus Tausendundeiner Nacht stammen ursprünglich aus Indien oder Persien oder verweisen explizit auf diese Orte. Zum Ursprung, der Zusammenstellung und der europäischen Verbreitung dieser riesigen Sammlung vgl. Robert Irwin: The Arabian Nights: A Companion. London 1994, und verschiedene Essays in Ulrich Marzolph [et al.] (Hg.): The Arabian Nights Encyclopedia, 2 Bde. Santa Barbara, CA 2004. Für eine Illustration aus dem 18. Jahrhundert, welche die islamische Präsenz überall in Asien betont, vgl. Locke: Music and the Exotic (Anm. 2), 61-62 (Abb. 3.5).

50 Für weitere solche Fälle wie beispielsweise die Titelrollen in Händels Serse, Tolomeo und Tamerlano, sowie Tolomeo in Giulio Cesare, vgl. Locke: Music and the Exotic (Anm. 2), 250258, 261-266. Locke: Alien Adventures (Anm. 1). 
einer fernen Kultur zugeschrieben wurden, schützte man sich davor, dass ein Vertreter der Regierung, der Kirche, Rezensenten oder jemand im Publikum sie als Spitze gegen heimische Fürsten oder politische, religiöse oder soziale Trends ,zu Hause' verstehen konnte.

Wer Repräsentationen des exotischen ,Anderen' in der westlichen Kultur untersucht, sollte die Operngeschichte berücksichtigen. Nur wenn wir uns mit den Details dieser Werke genau befassen, können wir verstehen, welche Bedeutung ein Werk in seiner eigenen Zeit hatte - nicht zuletzt, wie darin vorherrschendes Wissen (oder ,Wissen') sowie zeitgenössische politische Debatten, ethnische Vorbehalte und Gender-Vorurteile verarbeitet wurden. Auch im Hinblick auf seine heutige Bedeutung ist dies relevant. Schließlich haben die Fragen, um die es auf der Opernbühne damals ging - dazu gehören die grundlegenden menschlichen Spannungen zwischen der individuellen Erfüllung und der sozialen Verantwortung, aber in einigen Werken auch die besonderen Spannungen zwischen Nationen, Kulturen, Religionen, Osten und Westen et cetera - weiterhin eine große Bedeutung für unser Leben und unser Handeln in einer modernen, immer stärker globalisierten Welt. ${ }^{51}$

51 Vgl. Ralph P. Locke: Musicology and/as Social Concern: Imagining the Relevant Musicologist. In: Nicholas Cook und Mark Everist (Hg.): Retbinking Music. Oxford 1999, 499-530. 